\title{
Are nominal same-different matches slower due to differences in level of processing or to response competition?
}

\author{
CHARLES W. ERIKSEN and WILLIAM P. O'HARA \\ University of Illinois, Urbana-Champaign, Champaign, Illinois
}

\begin{abstract}
Eriksen, O'Hara, and Eriksen (1982) have proposed that the latency advantage of same over different judgments when the match is based upon physical identity is due to differential amounts of response competition between the responses by which the judgment of same or different is signified. Responses of "different" are slowed by a high level of priming in the competing response signifying same. In the present experiment, the response competition model is extended to nominal matches and in particular to what Proctor (1981) has termed the "name-physical disparity" - a pair of letters are more rapidly judged to have the same name if they are the same case (e.g., a a) than if they are in different cases (e.g., A a). While response competition effects were found to occur in nominal matches of this kind, the name-physical disparity was greater than could be attributed solely to response competition. Evidence was obtained that part of the name-physical disparity could be attributed to the subject's having two chances to make a nominal match when the letter pair was identical both physically and in name. The match could be made either on the basis of the physical or the name code. It was assumed that name and physical codes were processed at least partially independently.
\end{abstract}

Matching tasks in which a subject is asked to determine whether two stimuli are the same or different are increasingly used for the study of cognitive problems. It has become obvious, however, that the usefulness of the task is limited by our meager understanding of the processes by which a person arrives at such a judgment. Accordingly, there has been increased research oriented toward explicating the operations by which a person performs this most basic discrimination. Alphanumeric characters quite frequently are used as stimuli in these experiments, and letters permit the match to be made not only upon the basis of physical identity, for example, AA, but also on the basis of name identity, for example, Aa.

There have been two salient findings from this research effort. Judgments of same typically are made with a shorter latency than are judgments of different (see Krueger, 1978), and when the subject is required to match on the basis of name identity, the judgment that the target stimuli have the same name is more rapid when the stimuli are physically identical than when one of the targets is the upperand the other is the lowercase version of the letter (Posner \& Keele, 1967; Proctor, 1981).

Proctor (1981) has proposed an extensive and interesting model that provides an account of the pro-

This research was supported by Public Health Service Research Career Program Award K6-MH-22014 to the first author and by USPHS Research Grant MH-01206. The authors' address is: Department of Psychology, University of Illinois, UrbanaChampaign, 603 East Daniel, Champaign, Illinois 61820. cesses that produce these results. The difference in latencies between same and different judgments is attributed to response competition between name codes. When two different letters are presented to the subject, they are presumed to activate two name codes. The simultaneous activation of two codes is assumed to result in mutual inhibition and thus a delay in their availability to the subject. The "namephysical disparity," as Proctor has termed the latency differences obtained with physically same as opposed to name-same stimuli, is accounted for in terms of differences in levels of processing. When the stimuli are physically identical, the match is made on the basis of a physical code rather than the name code that is required when the stimuli are only name identical. Physical code matches are assumed to occur at an earlier level of processing and to be available more rapidly than name codes.

Recently, C. W. Eriksen, O'Hara, and B. A. Eriksen (1982) demonstrated the role of response competition in simultaneous same-different matches. They assumed a continuous flow model of visual information processing (C. W. Eriksen \& Schultz, 1979) and proposed an account of simultaneous matching based upon response competition between the two responses the subject uses to signify his judgments. While Proctor's model recognizes response competition in terms of name codes, the C. W. Eriksen et al. model draws upon recent findings with response competition effects (B. A. Eriksen \& C. W. Eriksen, 1974; C. W. Eriksen \& B. A. Eriksen, 1979; C. W. Eriksen \& Hoffman, 1973; 
C. W. Eriksen \& Schultz, 1979; Keren, O'Hara, \& Skelton, 1977) and proposes that response competition exists not only between name codes but also between the responses the subject uses to signify his judgment of same or different.

In terms of the continuous flow conception of visual information processing, the visual percept develops gradually over time. As energy is integrated by the visual system, the percept develops from an initial amorphous blob to increasingly fine representation of detail. Activation of recognition and other responses does not await full development of the percept. Instead, as the percept develops, priming flow to relevant responses begins with the priming flow becoming increasingly restricted to those responses that are still compatible with the developing percept. A primed response is released when the priming reaches a response criterion. The speed with which a response is executed depends upon the number of competing responses that are primed and the level of prime that they have achieved. (See Grice, Nullmeyer, \& Spiker, 1982, for a very similar processing model.)

The response competition model of simultaneous matching tasks attributes the longer latency for different judgments to a greater degree of response competition when the stimuli to be matched are different. Most stimuli employed in matching experiments have many characteristics or features in common, even when the target stimuli are different. They most generally are the same size and, if letters, frequently will have curves, angles, and straight lines in common. Furthermore, at early levels of percept development, fine detail is not available and the percepts of the target forms, even for grossly different stimuli, will have similarities.

A comparator process is assumed to begin detecting similarities and differences early in percept development. The similarities that are detected prime the response signifying same. By the time the percept is developed far enough so that differences can be confidently detected, the level of priming built up for the same response is sufficient to inhibit and slow the execution of the response signifying different. When the target stimuli are physically identical, the comparator process does not detect any differences except on those occasional trials in which random noise perturbation occurs in the sensory perceptual system (Krueger, 1978). As a consequence, the priming in the same response, on the average, reaches the evocation threshold, or criterion, with a low level of prime in the competing response that signifies different.

In general, judgments of same will require a higher, or more complete, development of the percept than will judgments of different, particularly if the stimuli to be matched are not grossly disparate. The criterion for a same response is assumed to be the subject's confidence that the percept has developed fully enough to contain sufficient detail to reveal any differences that might exist. The criterion for a different response, on the other hand, need be only the confidence that a difference has been detected.

Since the correct judgment of same depends upon a high level of percept development, it might be expected that same judgments would be slower than different judgments, but the latency differences of same and different judgments, as measured in our experimental settings, generally are in the reverse direction. This results from the greater response competition that exists for the execution of a different response. On many trials, the subject's response signifying different (vocalization, leverpress, etc.) is slowed in its execution by the priming that exists in the competing response signifying same; responses signifying same are executed with little or no inhibition from the competing different response.

The Eriksen et al. (1982) experiment found strong evidence for response competition when the subjects matched letter stimuli on the basis of physical identity. The degree of priming of same and different competing responses was manipulated by the use of an extraneous noise letter in the display that varied in its similarity or featural overlap with the target letters. For correct same judgments, no reliable latency differences were obtained between the noise and the no-noise control conditions when the noise letter was identical to the targets. When the noise letter had only partial feature overlap with the targets, the latency for same judgments was longer than that for different judgments. Latency for same judgments increased further when the noise letter was made even more dissimilar to the targets.

The latency of different judgments was also found to respond to noise-letter and target similarity. Priming of a competing same response by making the noise letter identical to one of the target letters increased different response latencies over the condition in which all three letters in the display were dissimilar.

Similar results have been obtained by other investigators who introduced noise stimuli into the displays for same-different discriminations. Krueger (1973) used noise consisting of underlines and plus and equal signs. When these noise symbols were different for each member of the target pair of letters, the latency of same trials was increased. In another study, Krueger (1970) found that when the noise was similar for each member of the target pair, latency on different trials was increased.

Although Krueger did not interpret his results in terms of competition between the responses signifying same and different, Keren, O'Hara, and Skelton (1977) made explicit use of a concept of response competition in their experiments designed to determine the depth of processing of the noise stimuli. 
In different experiments, these investigators required subjects to match pairs of letters on the basis of physical identity, nominal identity, and category membership (vowel or consonant). A pair of identical noise letters was presented simultaneously with the target letters in the display. These noise letters were presented in an irrelevant display position, and their relationship to the target pairs was varied systematically. The noise letters could be physically identical to the targets, be identical in name or category, or could mismatch the targets at each of these levels. The presence of the noise letter slowed reaction time to the targets, but the amount of interference was a function of the relationship of the noise to the targets.

On same, trials, reaction times were markedly increased if the noise letters were incompatible with the targets at the level of match. On different trials, reaction times were significantly longer if the noise letters matched one of the target members than if the noise letters were different from either target in name and form.

The results from both Krueger's experiments and those of Keren et al. are consistent with the continuous-flow/response-competition model of samedifferent discriminations. The question may be raised, however, as to whether these response competition effects are present in the standard samedifferent discrimination in which noise stimuli are not presented in the display along with the targets. If the comparison process was holistic, response competition could be obtained when noise letters were introduced in the display but be absent when only the target members were presented. For example, if, on a different trial, the targets $A$ and $E$ were presented with the noise letter $E$, comparing the letters as wholes would prime the response signifying same whenever the target $\mathrm{E}$ was compared with the noise $\mathrm{E}$. But if only the targets were present in the display, a holistic comparison of the $\mathrm{A}$ and $\mathrm{E}$ would not lead to the priming of a competing same response. Only if the comparison process is in terms of subunits, such as features or characteristics, would there be a basis for detecting similarities that could lead to the priming of the competing same response.

The C. W. Eriksen et al. (1982) paper strongly suggests that matching is not at the holistic level. Rather, the comparison is at a feature or characteristic level, as shown by their finding that the latency of correct same responses increases with increasing featural mismatch between the noise letter and the targets. The response competition model assumes that the stimuli are matched or compared in terms of subunits such as features or characteristics. It assumes, further, that as these features become available in the developing percept, they are not immediately collated with spatial position. In other words, features, and even identification, of stimuli are processed at least partially independently of spatial position information. This assumption is supported by the findings of Estes, Allmeyer, and Reder (1976), Treisman and Gelade (1980), and Wolford (1975). The response competition model further assumes that as similarities or differences are detected in the developing percept, they prime the relevant response by which the subject signifies his or her judgment. As processing continues, these features are collated with spatial position, but the priming they have contributed to the associated response does not decay immediately. Thus, if a similarity in features between a target and a noise stimulus has been detected, the priming that this detection has contributed toward the response signifying same is not immediately dissipated when position information becomes available.

The purpose of the present study was to extend the response competition interpretation to nominal matches and, in particular, the "name-physical disparity." Although the C. W. Eriksen et al. (1982) experiment investigated only physical identity matches, response competition would appear to play a large role in the "name-physical disparity." When the target stimuli have the same name but are physically nonidentical, the comparison process will detect the physical differences in the two stimuli as the percept develops. The detection of these differences in turn would prime the response for different. Thus, with name-identical targets, correct responses signifying same are made with a high degree of priming of the competing response signifying different. In contrast, physically identical pairs do not prime the competing different response, except for occasional random noise in the sensory perceptual system, with the result that the response signifying same is not slowed in its execution by the inhibition of a primed response signifying different.

Although a concept of response competition has an important status in Proctor's model, it is restricted to competition and mutual inhibition between name codes when different stimuli are presented. He does not apply the competition concept to an explanation for the "name-physical disparity." Instead, Proctor attributes the "name-physical disparity" to a difference in the level of processing. Physically identical stimuli are matched more quickly when a name match is required because the match is carried out at the level of a physical rather than a name code.

Response competition and a difference in level of processing are not mutually exclusive explanations for the "name-physical disparity," but response competition would appear the more parsimonious since it explains both the physical disparity and the "name-physical disparity" in terms of a single construct. A major purpose of the present experiment was to determine if the "name-physical disparity" 
could be accounted for solely in terms of response competition or whether other factors were involved.

Should the "name-physical disparity" turn out to be greater than can be attributed solely to response competition, then it is possible that there is an explanation other than Proctor's level-of-processing one. Physically identical stimuli can be matched on the basis of either the physical code or the name code. Name-same stimuli can be matched only on the name code. In terms of the continuous flow conception of information processing, the developing percept primes relevant naming responses in addition to relevant physical codes. If the activation of these codes is independent or even partially independent and the distributions of their latencies for activation overlap, then on a certain proportion of trials the name code will be available before the physical code and the latency at which one or the other code will be available will average less than the average latency for either. The latency advantage that a subject would have from being able to use either code on the match would be dependent upon the degree of overlap of the distributions of processing time for the two codes and the degree to which their activation or processing was independent. The viability of this "two chances" hypothesis requires evidence that name codes are activated on some proportion of the trials even when the stimuli to be matched are physically identical. The second purpose of the present experiment was to look for such evidence.

\section{Design}

In the present experiment, the letters $H$ and $D$ in upper- and lowercase versions constituted the target set. Subjects were asked to respond "same" if the letters had the same name. The subjects indicated their judgments of same or different by moving a small lever to the left or to the right. In addition to the target pair of letters, which occurred in predesignated positions in the display, each display contained a noise stimulus. The noise either was chosen from the target set of upper- and lowercase letters or was one of two nonsense forms, $F_{1}$ and $F_{2}$ (see Figure 1). After completing two experimental sessions, the subjects were given extensive training in labeling the nonsense forms. Half of the subjects learned to call $F_{1}$ by the name " $H$ " and $F_{2}$ by the name "D." For the other subjects, the assignment of labels to the nonsense forms was reversed. Following training, the subjects completed two more sessions that were essentially replications of the first two.

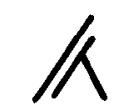

Form 1

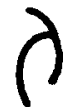

Form 2
Figure 1. The two nonsense forms used as noise stimull.
In this design, when the targets are physically identical, the noise stimulus can be physically the same (same letter and case as the targets), name the same (alternate case version of the targets, e.g., $\mathrm{H} \mathrm{h} \mathrm{H}$ ), the other target letter in either case (e.g., $\underline{\mathrm{H}} \mathrm{d} \underline{\mathrm{H}}$ ), or a nonsense form. With the exception of physically identical noise, comparable noise conditions exist for name-same target pairs. (In these display illustrations the target members are underlined.)

The presence of response competition in this paradigm can be tested by comparing physically same stimuli with identical noise $(\underline{h} h$ h) with the namesame noise condition (h $\mathrm{H} h$ ). According to the response competition theory, the presence of the alternate-case version of the target letters in the display should, through its dissimilarity in features, prime the response signifying different, which will inhibit and slow the execution of the response "same." Thus, the same-name noise condition should lead to significantly longer latencies for "same" judgments than would identical noise when the stimuli are physically the same.

This design also permits testing to determine whether or not response competition is sufficient by itself to account for the "name-physical disparity." The test is a comparison of the physically same targets with name-same noise (e.g., $\underline{\mathrm{h}} \mathrm{H}$ h) with namesame targets and name-same noise (e.g., $\underline{h} h \mathrm{H}$ ). According to the response competition model, at least part of the "name-physical disparity" is due to the physical differences in name-same stimuli priming the response signifying different, which inhibits and slows the execution of the response signifying same. By adding to the display, as noise, the alternate-case version of the identical target pair, we have produced essentially the same amount of dissimilarity in the display for a physically same match as exists for the name-same match. For a name-same noise condition, the same physical stimuli are present in the display whether the targets are identical physically or only in name and should lead to comparable priming for the response signifying different. If the "name-physical disparity" can be attributed solely to response competition, average latency for physicall: same targets with name-same noise should not differ significantly or appreciably from name-same target: with name-same noise.

As pointed out above, there is reason to believ that the "name-physical disparity" may involv more than response competition. Both Proctor'. levels of processing and the "two chances" hypothesis presented above may be involved. The "tw' chances" hypothesis requires evidence that name codes are involved on some proportion of the trials when the match is for physically same stimuli. The present design provides a test for such evidence at two levels. If physically identical targets are matchec solely on a physical code, then whether the noise 
stimulus has the same name as the targets or not should be immaterial. Interference from the noise stimulus should result only from priming of the different response due to physical dissimilarities between the noise and the target. If, however, a name code is involved in physically same matches, at least on some proportion of the trials, a noise stimulus with a name different from that of the targets should interfere more than noise that has the same name as the targets or is an as yet unlabeled nonsense form. Response competition would exist between the different name codes as well as from competing response priming arising from physical dissimilarities. As Proctor (1981) proposes, the name-different noise would activate a name code that was different from that evoked by the targets, resulting in mutual inhibition and delay in the availability of the name code for the targets. Inhibition between name codes would reduce the advantage that a subject would have for physically same matches that would arise on the basis of the "two chances" hypothesis.

While the finding of longer latencies with namedifferent noise than with name-same noise on physically same matches would suggest that name codes are involved when the match involves physically identical stimuli, the evidence would not be unequivocal. Comparisons of name-same, name-different, and nonsense noise can provide only suggestive evidence as to the role of name-code conflict inasmuch as the comparisons are confounded by possible differences in feature overlap or the similarity between these noise stimuli and the targets. Since we have no absolute measure of feature overlap of the noise with the targets, it is possible that any obtained latency differences are due to the differences in the physical features contributing in varying amounts to the priming of the motor response signifying different rather than to a conflict in eliciting name codes. For example, one could plausibly argue that name-same noise displays such as $\underline{h} H \underline{h}$ and $\underline{d} D \underline{d}$ are more physically similar than noise-different displays such as $\underline{\mathrm{h}} \mathrm{d} \underline{\mathrm{h}}$ and $\underline{\mathrm{d}} \mathrm{H} \underline{\mathrm{d}}$. Thus, the longer latency of the latter may be due to greater priming of the lever movement for different due to the feature differences rather than to the name code conflict.

An evaluation of noise name unconfounded by featural differences can be made by analyzing the effect of the nonsense-form noise after letter names have been associated with these forms. In the second part of the experiment, the same nonsense forms could be either name identical or name different for the same pair of targets. If name codes are activated when the match involves physically identical targets, we would expect that the latency would be longer for a nonsense form with a learned name that was different from that of the targets than for a nonsense form that had a learned name that was the same as that of the target.

\section{METHOD}

\section{Subjects}

Eight University of Illinois students (four men) served as paid volunteers. Each was right-handed and had normal or correctedto-normal vision.

\section{Apparatus and Stimuli}

The stimulus displays contained two target letters and either a letter distractor or a nonsense form, all arranged along a horizontal line. The target letters appeared in the extreme right and left positions while either the letter or nonsense-form noise element occupied the center position. The three positions were equally spaced, with $2.2 \mathrm{deg}$ of visual angle separating the centers of the two target letters. The target letters were Zip-a-Tone Futura demibold 24 didot point letters. They were black and appeared against a white vinyl card background. The letters $H, h, D$, and $d$ were used as target letters and as noise letters as well. Each was used equally often. The nonsense symbols were constructed using letter parts taken from the same Zip-a-Tone character letter type used for the letters, with the restriction that each nonsense form be approximately the same height and width as a capital letter from that set. The two nonsense forms are shown in Figure 1.

The stimulus displays were presented in a Scientific Prototype three-channel tachistoscope equipped with Sylvania F4 TS/CWX lamps. Luminances of the white backgrounds for all fields were set at $4.5 \mathrm{fL}$. The subjects responded by moving a small lever to the right or the left to indicate their judgments of same or different. Reaction times were recorded to the nearest millisecond on a Hunter Model 1522 digital Klockounter. During the fourth experimental session, a microphone and an audio threshold detection relay were used to trigger the timer so that naming latencies could be recorded.

\section{Procedure}

The subjects were instructed to move the lever to designate same whenever the targets were name-identical. Target matches for same responses were either physically identical or nameidentical. Another variable for same trials was the relation of the noise element and the target letters. When the target letters were physically identical, the distractor letter could be identical (e.g., $\underline{\mathrm{H}} \mathrm{H} \underline{\mathrm{H}}$ ), name-same (e.g., $\underline{\mathrm{H}} \mathrm{h} \underline{\mathrm{H}}$ ), or name-different (e.g., $\underline{H} \mathrm{D} \underline{\mathrm{H}}$ ). In addition, one or the other of the two nonsense forms was used as the noise element on a certain number of trials. Whether the names of these nonsense forms were the same as or different from those of the target letters was not determined until Session 4, after which the first half of the experiment was repeated. When the target letters had the same name, the noise letter could have either the same name, and thus be physically identical to one of the target letters (e.g., $\underline{H}_{\mathbf{h}} \mathbf{h}$ ), or a different one (e.g., $\underline{D}$ h g). Also, similar to the physically identical trials, each of the nonsense forms was used as the noise element with name-same target pairs.

The name-different displays (c.g., $\underline{\mathrm{H}} \mathrm{d}$ D) consisted of a factorial combination of different target pairs with each of the noise items. For the purposes of final analysis, three major target-noise conditions were recognized: (1) the noise element was physically identical to one of the target elements (e.g., H H D); (2) the noise element was name identical to none of the target elements (e.g., $\underline{H}$ h D); and (3) the noise element was one of the nonsense forms. The subjects were told to initiate each trial, by depressing a pushbutton held in the left hand, after they had clearly focused upon the fixation cross located in the center of the fixation field. The fixation field was replaced by a blank white field, which remained on for $150 \mathrm{msec}$. This was followed by the stimulus display, which remained on for $150 \mathrm{msec}$. Then the fixation field reappeared.

The subjects were instructed to compare the names of the two target letters and to move the response lever to the left (right) if the targets had the same name and to the right (left) if the names were different. It was stressed to the subjects that they 
were to ignore the letter or nonsense form between target letters.

The subjects were told to respond as quickly as possible while attempting to maintain a low error rate. Error trials were rerun in a random fashion later in the experimental session. The subjects were given reaction-time and accuracy feedback after each trial.

Each subject participated in six sessions. The first session was practice, and the data collected from this session were excluded from analysis. Sessions 2, 3, 5, and 6 were all identical except that Sessions 5 and 6 occurred after the subject had had a session during which he learned names for each of the two nonsense syllables. In each of the four sessions, the subject viewed 144 stimulus displays representing the different conditions. There were an equal number of displays containing same and different target pairs. Of the 72 same displays, 40 consisted of physically identical targets. These $\mathbf{4 0}$ displays consisted of 8 each of the three different combinations of noise and target letters and 8 each with the two nonsense forms. The remaining 32 same displays consisted of name-identical targets. They, in turn, consisted of 8 displays of each of the combinations of target and noise letter and 8 with each of the nonsense forms.

Session 4 was devoted to learning names for the nonsense syllables. During this session, half of the subjects, selected randomly, learned to call $F_{1}$ " $D$ " and $F_{2}$ " $H$." The nonsense-form/ name combinations were reversed for the other half of the subjects. The session began with several minutes given the subjects to study the two form-name combinations. This was followed by 40 practice trials during which the forms were displayed tachistoscopically and naming latencies were recorded using a voice key. The voice key was utilized instead of a lever movement to avoid any response competition with lever responses made throughout the rest of the experiment. After the practice trials, each subject received 120 experimental letter-naming trials. Sixty of these trials were nonsense-form trials and the others were divided equally among the upper- and lowercase versions of the target letters. Naming latencies for these trials were recorded, and it was found that, on average, naming latency for the two nonsense forms was 107 msec longer than it was for the letters.

At the beginning of each of the same-different experimental sessions, the subjects were given 20 practice trials. In addition, at the beginning of Sessions 5 and 6 , each subject had 40 practice trials during which he or she named the nonsense forms using the learned letter designation.

\section{RESULTS AND DISCUSSION}

\section{Errors}

The number of errors in judgments was too low to permit meaningful analysis. Consistent with previous studies in which we introduced noise elements into the matching task (Eriksen et al., 1982; O'Hara 1977), there was no evidence of a speed accuracy tradeoff in the data. Across experimental conditions, mean errors and latencies correlated .27.

\section{Same Trials}

Latencies were evaluated in a four-way ANOVA (subjects, physically same vs. name-same trials, noise conditions, and sessions). Since the identical-noise condition could not occur in the name-same trials, it was not included in the analysis. All the main effects were significant $(p<.01)$, as was also the interaction between noise conditions and physically same vs. name-same matches. The significant effect of sessions represents practice. Overall, latencies for the last two experimental sessions averaged $13 \mathrm{msec}$ faster than for the first two sessions. However, this practice effect did not interact with any of the other experimental variables $(p>.3)$. Subsequent analyses involving comparisons of pairs of means used a t test based upon the appropriate error variance from the ANOVA. ${ }^{1}$

In Figure 2, latencies have been averaged over subjects for the first two sessions and are plotted as a function of the noise condition for physically same and for name-same targets. Since the data are from the first two experimental sessions, the nonsenseform noise data are from before letter names were associated with each of these two forms. It is notable that the identical, name-same, and name-different noise conditions are quite comparable to the conditions employed in Experiments 2 and 4 of the Keren et al. (1977) study. For both physically same and name-same targets, the results shown in Figure 2 are strikingly comparable to those obtained by those investigators.

The variation in judgmental latency for physically same and name-same targets as a function of noise condition reflects the compatibility or incompatibility of the noise and targets at the feature and name levels. The most direct manifestation of incompatible noise at the feature level is obtained by comparing physically same targets and identical noise (e.g.,

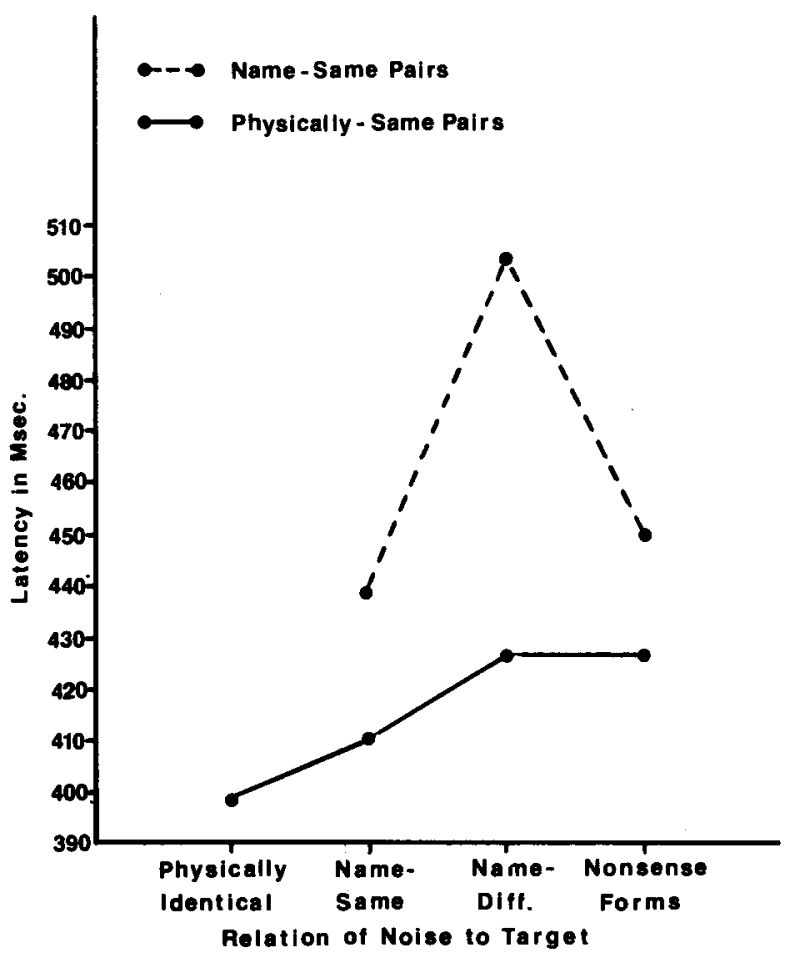

Figure 2. Mean latency of correct same responses as a function of the relation of the nolse letter to the target letters. The functions are plotied separately for the trials on which the targets were physically identical and for the trials on which they were name identical but physically different. 
$\underline{\mathrm{H}} \mathrm{H} \underline{\mathrm{H}}$ ) with physically same targets and name-same noise (e.g., $\underline{\mathrm{H}} \mathrm{h} \underline{\mathrm{H}}$ ). When the noise letter is the alternative case of the target pair, reaction-time latency is increased by $11 \mathrm{msec}$ over that when the target and the noise letter are physically identical $(p<.05)$. This compares well with latency differences of 10 and $16 \mathrm{msec}$ obtained by Keren et al. (1977) in comparable comparisons made in their Experiments 2 and 4.

Under all noise conditions, physically same matches are made with a shorter latency than name-same matches, a finding that accords generally with the literature (see Proctor, 1981). Proctor's model accounts for this disparity in terms of the level of processing at which the match is made. Physically same stimuli are assumed to be matched at the physical code level, which is processed more rapidly than the name codes required for nominal matches. One purpose of the present experiment was to determine if it was necessary to posit a difference in level of match or whether the difference in latency of physically same and name-same matches could be accounted for solely in terms of response conflict.

The most appropriate comparison for physically same and name-same performance is when both types of targets have a name-same noise letter in the display (e.g., for physically same, $\underline{\mathrm{h}} \mathrm{H} \underline{\mathrm{h}}$, and for namesame displays, $\underline{h} h \underline{H})$. With name-same noise letters, the overall dissimilarities or featural differences in the display are identical. The displays differ only in the location of the upper- and lowercase versions of a letter. Thus, both types of displays would seem to give an equivalent amount of priming to a different response that arises solely from featural differences. The displays with the physically same targets, however, have a latency shorter than that for the nominal match $(\mathrm{p}<.01)$. This finding is consistent with Proctor's theory that the match is made in terms of physical codes when the targets are physically the same. However, the previously noted significant difference obtained between physically identical noise and the name-same noise condition with physically same targets shows that featural dissimilarity and, thus, response conflict are also contributing to the "name-physical disparity."

There is evidence, however, that the name code level is involved at least on a certain proportion of physically same matches. With physically same targets, name-different noise is significantly longer than name-same noise $(p<.01)$. However, this evidence is not unequivocal. As shown in Figure 2, the latency for name-different noise is essentially the same latency as for nonsense-form noise. In the latter instance, the name conflict would not be expected, since these data are from the first half of the experiment before letter names were associated with the nonsense form.

Comparisons of name-same, name-different, and nonsense-noise conditions can only be suggestive evi- dence of the role of the name-code conflict inasmuch as the comparisons are confounded by possible differences in feature overlap or similarity of these noise stimuli with the target. Since we cannot equate absolutely the similarity or featural overlap of these noise letters and nonsense forms with the targets, it is possible that latency differences are due to different amounts of priming of the motor response signifying different arising from featural differences rather than to conflict in name codes that are elicited.

An evaluation of the effect of noise name unconfounded by featural differences can be made by analyzing the effect of the nonsense-form noise after letter names have been associated with these forms. In Figure 3, average judgmental latencies for same responses are shown for physically same and namesame targets when the nonsense-form noise had a learned name that was either the same as that of the targets or different. The data are shown for before and after training with the nonsense forms. Differences within physically same and name-same matches in the before condition are due to individual differences, since the assignment of the letter name to a nonsense form for a given subject was not matched on the basis of that subject's average latency for that nonsense form before the learning session. Also shown in the figure is the average latency for same judgments to physically same and name-same targets under the name-same noise condition obtained in the first two (before) and the last two (after) experimental sessions. These latter data are included to provide a reference point for the practice effects.

The nonsense-form data in Figure 3 were analyzed in a four-way ANOVA (subjects, before and after training, noise name same vs. name different, and physically same vs. name-same targets). The effect of nonsense-noise name was significant $(p<.02)$, as was also the interaction between before and after training and nonsense-noise name $(p<.01)$.

For both physically same and name-same targets, judgmental latencies are longer if the nonsense-form noise has a letter-name different from that of the targets. It would appear from the figure that the effect of name congruity between the targets and the noise was primarily in terms of facilitating judgments when the targets and the nonsense noise had the same name. However, this appearance is misleading. There is a significant practice effect between the first and second half of the experiment which, averaged over conditions, amounted to $13 \mathrm{msec}$. The data for the case in which the noise letter had the same name as the targets, as shown in Figure 3, provide a reference comparison for this practice effect.

When this practice effect is taken into account, the data in Figure 3 are seen to show that the namedifferent conditions did not benefit from practice, whereas the name-same conditions show a practice effect. In other words, if the nonsense form had a 


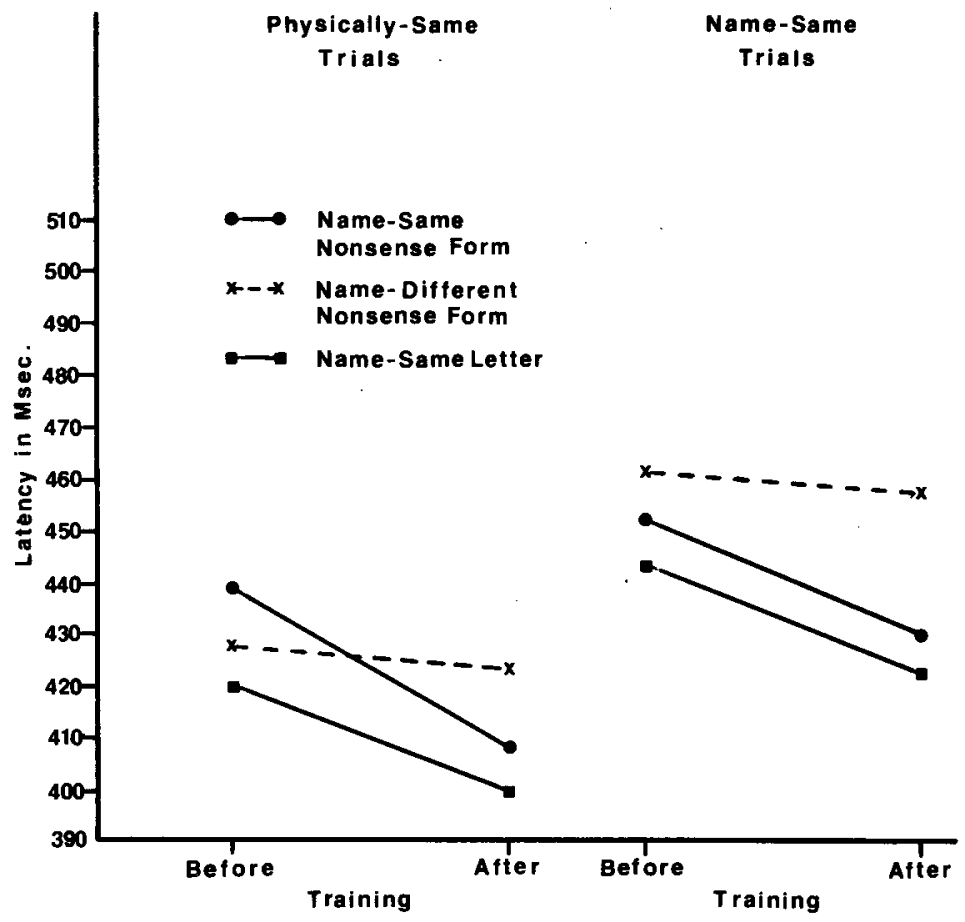

Figure 3. Mean latency of correct same responses when a nonsense form was included as noise in the display. In the after-training condition, the nonsense form had a learned name either the same as that of the target letters or different. Data for the nume-same letter condition (the nolse was a letter with the same name as the targets') are provided as a reference for practice effects.

learned name that was the same as that of the targets, little impairment was evident. The gain in performance was comparable to the practice effect shown for a noise letter that had the same name as the target. However, when the nonsense form had a learned name that was different from that of the targets, interference was apparent in the judgment latency, since the latency was approximately $13 \mathrm{msec}$ longer than would be expected from the practice effect. These results are quite clear in showing that name codes are involved even in physically same matches when the possibility of featural differences are controlled.

We had expected a greater effect of noise and target-name compatibility for name-same targets than for physically same ones. This expectation was based upon the reasoning that while physically same targets can be matched on the basis of the physical code, name-same targets require the name code for matching and therefore noise-target name incompatibility should be more disruptive. As Figure 2 shows, the noise name-different condition produces considerably more impairment in response latency for name-same targets than it does for physically same targets. The failure to find a comparable interaction with the letter-labeled nonsense forms is probably attributable to the necessarily restricted practice the subjects had in assigning letter names to these forms. Even though an experimental session was devoted to labeling practice, it still took subjects approximately $100 \mathrm{msec}$ longer to respond with a letter name when a nonsense form was presented than to respond with a letter name when a letter was presented. Eriksen and Schultz (1979) have found that delaying the presentation of an incompatible noise letter by $100 \mathrm{msec}$ significantly attenuates its response competition effect. If physically delaying a noise letter is comparable to the increased latency in its availability as a response, then we could expect the labeled nonsense forms to be a less sensitive measure in the present experiment.

Our analysis of the data so far has shown that the "name physical disparity" cannot be accounted for completely in terms of response competition activated by featural differences among the target stimuli. That such featural differences are involved, though, has been supported by the significantly greater latency obtained for name-same noise than with identical noise with physically same targets, as well as by the findings of C. W. Eriksen et al. (1982) and Keren et al. (1977). The remainder of the "namephysical disparity" may well be due to the level of processing differences as proposed by Proctor (1981), but the results obtained with the labeled nonsense forms show that name codes are involved with physically same matches at least on a significant pro- 
portion of trials. This latter finding is consistent with our "two chances" hypothesis, namely that physically same stimuli can be matched on the basis of either a physical code or the name code, whereas name-same matches can be made only at the name code level.

The differences between the level of processing explanation and the "two chances" hypothesis are subtle, but important. The level of processing explanation implies that the physical code must precede the name code and that the name code is activated only upon completion of the physical code. These implications are unnecessarily restrictive. In our preference for a continuous flow type of information processing, we conceptualize the activation of name and physical codes as essentially parallel or independent processes. For naming responses or codes that are as overlearned as are letter names among college students, we would expect that the developing percept would be activating these name codes while it was activating the physical code. The physical code may on the average have an inherent speed advantage, but, as long as the distribution of these latencies overlap the distribution for the name code, there will be a certain proportion of trials on which the name code is available before the physical code is. The results obtained with the labeled nonsense forms support this conception.

While the evidence that name codes are involved at least occasionally on physically same matches supports our "two chances" hypothesis, it is not unequivocal. A sequential, or stage, conception of levels of processing is still viable if one assumes that subjects are occasionally inconsistent in their use of a physical code when it is available to them. Subjects may occasionally neglect to use the physical code for matching and await the more slowly available name codes, particularly when physically same and name-same matches are intermixed as trials in the same block.

The present data also bear upon the possibility of competition between, and mutual inhibition of, name codes. Proctor (1981) and Proctor and Rao (in press) make extensive use of this possibility as an explanation for the "name-physical disparity." C. W. Eriksen and Schultz (1979) discussed the possibility that internal recognition responses (name codes) could compete and mutually inhibit each other much as has been observed for overt responses. They obtained some evidence to support this possibility, as did C. W. Eriksen and B. A. Eriksen (1979). If recognition responses or name codes are subject to mutual inhibition and competition, as are motor responses, this would represent a concept of major importance to information processing theories, particularly in view of the extensive evidence that such internal recognition responses can be primed or fa- cilitated by antecedent events (Kirsner \& Craik, 1971; Neely, 1977; Posner \& Snyder, 1975; Warren, 1974). The evidence from the present experiment is consistent with such a view.

We can presume that the nonsense form did not activate name codes or labels in the first half of the experiment. Thus, the interfering effects they had upon same judgments when used as noise stimuli in the display was in terms of the activation of the response signifying different due to their feature dissimilarity to the target letters for both name-same and physically same displays. However, these physical feature dissimilarities remained constant when the subjects learned letter names for the nonsense forms. Following this learning, interference effects were greater when the nonsense form had a learned name that was different from the name of the targets than when the learned name was the same. This increased interference after label training is consistent with what would be expected if the learned name for the nonsense form was now competing with or inhibiting the availability of the name code for the target stimuli.

Further evidence is found in the pronounced interference effect that is obtained with name-same stimuli when the name of the noise letter is different (see Figure 2). Here the display is of targets consisting of the upper- and lowercase versions of a letter and the noise letter is a different letter (e.g., h $\mathrm{D} \underline{\mathrm{H}}$ ). The feature dissimilarity in this type of display seems comparable to that obtained when the upper- and lowercase targets are accompanied by one of the nonsense forms before a label is learned for the form. But the amount of interference or delay in the judgment of same is markedly increased for the former type display. This is what would be expected if competition for name codes occurred. With the name-different noise displays (e.g., h $\mathrm{D} \underline{\mathrm{H}}$ ), two name codes are activated which presumably inhibit each other and thus delay their availability. Since the targets are featurally dissimilar, the subject would depend upon the name codes in order to make a correct same judgment. Before the learning of labels, the nonsense-form noise presented with same targets would evoke only a single name code, thus accounting for the much shorter latency with this type of display.

Both C. W. Eriksen and Schultz (1979) and Proctor (1981) have suggested that the competition between name codes delays their availability or access into consciousness. It should be noted that the present results can be accounted for without assuming that competition or inhibition occurs this early in processing. It is possible that a display that activates two different name codes produces both codes in consciousness either simultaneously or serially and that the two different name codes then act to prime 
the response signifying different in the same manner that featural similarities do. In this case, when the subject is presented with a name-same display such as $\underline{h} \mathrm{D} H$, both name codes $\mathrm{h}$ and $\mathrm{D}$ occur and, as a result, this difference in name adds to the priming of the response signifying different, which has already been primed by the feature dissimilarity in the display. This increase in priming can then account for further inhibition and delay of the judgmental response signifying same. This possibility cannot be ruled out by the present data.

\section{Different Trials}

So far in our analysis of the data, we have been concerned only with same judgments. The response competition model predicts not only that same judgments will be inhibited by featural or name code differences that are activated by the display, but also that the response signifying different will be inhibited and therefore delayed by similarities in the display. In the present experiment, different trials can be meaningfully classified into three types which differ in the degree to which they would be expected to prime a response signifying same. When the noise stimulus is a letter, there are two kinds of different displays, one in which the noise is physically identical to one of the targets (e.g., $\underline{H}$ H D) and the other in which the noise and targets are physically different (e.g., $\underline{H} \mathrm{~d} \underline{\mathrm{D}}$ ). The first of these displays has a higher degree of featural similarity than does the latter. Response competition would be expected to be greater in the first type, since the identity of two of the letters would prime a response signifying same and this priming would inhibit and delay the execution of the response signifying different.

The results support this expectation. Mean latencies for displays in which the noise and one target letter were identical were 520.6 and $492.7 \mathrm{msec}$ for the heterogeneous different displays. This difference is significant beyond the .01 level. The other type of different display was that in which a nonsense form occurred as the noise. Before label training, the nonsense form would constitute a display comparable to that in which all three letters were physically different. As such, the nonsense-form noise displays would be expected to perform in a similar manner. This was the case. The mean reaction time for different judgments when the noise was a nonsense form was $501 \mathrm{msec}$, which also was significantly shorter than that obtained in the different displays with two identical letters.

\section{REFERENCES}

Eriksen, B. A., \& Eriksen, C. W. Effects of noise letters upon the identification of a target letter in a non-search task. Perception \& Psychophysics, 1974, 16, 143-149.

Erikgen, C. W., \& Eriksen, B. A. Target redundancy in visual search: Do repetitions of the target within the display impair processing? Perception \& Psychophysics, 1979, 26, 195-205.

Eriksen, C. W., \& Hofrman, J. E. The extent of processing of noise elements during selective encoding from visual displays. Perception \& Psychophysics, 1973, 14, 155-160.

Eriksen, C. W., O'Hara, W. P., \& Eriksen, B. A. Response competition effects in same-different judgments. Perception \& Psychophysics, 1982, 32, 261-270.

Eriksen, C. W., \& Schultz, D. W. Information processing in visual search: A continuous flow conception and experimental results. Perception \& Psychophysics, 1979, 25, 249-263.

Estes, W. K., Allmeyer, D. H., \& Reder, S. M. Serial position functions for letter identification at brief and extended exposure durations. Perception \& Psychophysics, 1976, 19, 1-15.

Grice, G. R, Nullmeyer, R., \& Spiker, V. A. Human reaction time: Toward a general theory. Journal of Experimental Psychology: General, 1982, 111, 135-153.

Keren, G., O'Hara, W. P., \& Skelton, J. M. Levels of noise processing and attentional control. Journal of Experimental Psychology: Human Perception and Performance, 1977, 3, 653-664.

Kirsner, K., \& Craik, F. I. M. Naming and decision processes in short-term recognition memory. Journal of Experimental Psychology, 1971, 88, 149-157.

KRUEGE R, L. E. Effect of bracketing lines on speed of "same""different" judgment of two adjacent letters. Journal of Experimental Psychology, 1970, 84, 324-330.

KRUEGER, L. E. Effect of irrelevant surrounding material on speed of same-different judgment of two adjacent letters. Journal of Experimental Psychology, 1973, 98, 252-259.

Kruegen, L. E. A theory of perceptual matching. Psychological Review, 1978, 85, 278-304.

NeELY, J. H. Semantic priming and retrieval from lexical memory: Roles in inhibitionless spreading activation and limited-capacity attention. Journal of Experimental Psychology: General, 1977, 106, 226-254.

O'HarA, W. P. The spatial and featural effects of irrelevant letters on the speed of same-different judgments of two target letters. Unpublished master's thesis, University of Illinois, UrbanaChampaign, 1977.

Posner, M. I., \& Keele, S. W. Decay of visual information from a single letter. Science, 1967, 158, 137-139.

Posner, M. I., \& Snyder, C. R. R. Facilitation and inhibition in the processing of signals. In P. M. A. Rabbitt \& S. Dornic (Eds.), Attention and performance $V$. New York: Academic Press, 1975.

Proctor, R. W. A unified theory for matching-task phenomena. Psychological Review, 1981, 88, 291-326.

Proctor, R. W., \& RAo, K. V. Reinstating the original principles of Proctor's unified theory for matching-task phenomena: An evaluation of Krueger and Shapiro's reformulation. Psychological Review, in press.

Treisman, A. M., \& Gelade, G. A feature-integration theory of attention. Cognitive Psychology, 1980, 12, 97-136.

Warren, R. E. Association, directionality, and stimulus encoding. Journal of Experimental Psychology, 1974, 102, 151-158.

Wolford, G. Perturbation model for letter identification. Psychological Review, 1975, 83, 184-199.

\section{NOTE}

1. More conservative tests of significance could have been employed, but they were deemed unnecessary. With the exception of comparisons involving the nonsense forms, the comparisons of conditions in the present experiment closely replicate conditions obtained in Experiments 2 and 4 in the Keren et al. (1977) study, with nearly identical results.

(Manuscript received July 27, 1982; accepted for publication August 3, 1982.) 